\title{
Comparative Study of Clinical Outcomes of Anterior Cervical Discectomy and Fusion Using Autobone Graft or Cage with Bone Substitute
}

\author{
Jae Sung Ahn, June Kyu Lee, Joung Hun Kim \\ Department of Orthopedic Surgery, Chungnam National University School of Medicine, Daejeon, Korea
}

\begin{abstract}
Study Design: A retrospective study.
Purpose: To compare the clinical and radiological outcomes of autogenous bone graft and cage with bone substitute for anterior cervical discectomy and fusion.

Overview of Literature: The clinical outcomes of cage with bone substitute for anterior cervical discectomy and fusion is satisfactory.
\end{abstract}

Methods: Eighty four patients who underwent cervical spine surgery between February 2004 and April 2009 were includ ed. Fifty-nine patients were approached anteriorly and underwent anterior cervical discectomy and fusion by the SmithRobinson method (Group A), and 25 patients underwent fusion by decompression of the cervical spine and cage with bone substitute (Group B). We measured and evaluated the postoperative period until patients were able to ambulate, for pre- and postoperative symptomatic improvement, postoperative complications, pre- and postoperative change of lordosis, degree of endplate collapse or subsidence, and fusion rate and period of union.

Results: By Robinson's criteria, respectively 45, 10 and 4 patients in Group A experienced excellent, good and fair symptomatic improvement, and respectively 19, 5 and 1 patients in Group B experienced excellent, good and fair symptomatic improvement. The postoperative period in which patients became ambulant and the period of hospital stay was significantly shorter in Group B. Increase of lordosis at final follow up after surgery was significantly larger in Group A, as was the fusion period. Significantly more endplate collapse occurred in Group B.

Conclusions: Of patients who had anterior cervical discectomy and fusion, results of both groups were both satisfactory.

Key Words: Cervical vertbrae, Anterior cervical discectomy and fusion, Autobone graft, Cage, Bone substitutes

\section{Introduction}

Current surgical management for trauma and diseases of the cervical spine include the anterior approach, the posterior approach and the combined anterior and posterior approach. Anterior cervical disectomy and fusion of the cervical spine were first reported by Robinson and Smith [1].
Since then, they have become commonly adopted for the treatment of cervical lesions. Anterior fusion facilitates spinal cord decompression and bone union concomitantly and postoperative stability can be attained. It has therefore been used to treat lesions with excellent reported treatment outcomes [1-4].

Autogenous bone graft has previously been performed in anterior fusion. In recent years however, methods that entail

Received Nov 30, 2010; 1st Revised Jan 10, 2011; 2nd Revised Apr 6, 2011; Accepted May 12, 2011

Corresponding author: Jae Sung Ahn, MD

Department of Orthopedic Surgery, Chungnam National University School of Medicine,

640 Daesa-dong, Jung-gu, Daejeon 301-721, Korea

Tel: +82-42-220-7353, Fax: +82-42-252-7098, E-mail: jsahn@cnu.ac.kr 
the use of a cage - associated with side effects such as pain and complications at the donor site, disadvantages of autogenous bone graft, have been frequently used. To date, however, few studies have examined the clinical and radiological outcomes of these methods. In performing plate fixation via the anterior approach using a cage containing bone substitute and autogenous bone graft, we comparatively analyzed pre- and postoperative symptomatic improvement, postoperative complications, changes in pre- and postoperative lordosis curvature, surgical site endplate subsidence extent and the rate and period of union. In so doing, we attempted to evaluate the merits of both methods and their clinical effectiveness.

\section{Materials and Methods}

\section{Study patients}

For all patients who underwent cervical disectomy and fusion of the anterior and cervical vertebrae from February of 2004 to April of 2009, we excluded patients who underwent cervical spinal fixation of more than two segments, those who experienced vertebral body fracture due to trauma and those who could not maintain daily living due to underlying pre-operative conditions. In total, 84 patients were enrolled into the current study. Fifty-nine patients who underwent anterior cervical disectomy and fusion using autogenous bone were assigned to Group A. Twenty-five patients who underwent anterior cervical disectomy and fusion using a cage and bone substitute were assigned to Group B.

\section{Surgical methods}

Consistent with other types of anterior approach, a transverse incision was made along the skin to the target cervical spine segment. After approaching the site of injury, the spinal segment was confirmed by fluoroscopy. With the anterior expansion of the intervertebral disc space at the segment of the cervical area where there were fracture and disease using a Caspar retractor or a laminar distractor, the damaged intervertebral disc was removed. The anterior part of the lower endplate of the upper vertebral body in the superior segment, and the upper endplate of the lower vertebral body were grinded. This was followed by cortical bone removal. Subsequently, following the removal and grinding of the cartilaginous upper and lower endplates, on the cross section, the presence of a pinpoint bleeding was confirmed. In Group A, the struct bone was removed from the autogenous iliac bone and then prepared as a wedge-shaped struct bone. This was followed by insertion at an anterior height of 9-12 mm, and the posterior one which was shorter by 1-2 $\mathrm{mm}$ as compared with the anterior one. Thus, efforts were made to form cervical lordosis. In Group B, a cage (Cervios chronOS-synthetic B-tricalcium phosphate cancellous bone substitute, SYNTHES ${ }^{\circledR}$, West Chester, PA, USA) was inserted by using a casper retractor, where the insertion was performed by adding 3-4 $\mathrm{ml}$ of blood harvested from the bone marrow of the cervical vertebral body. Thereafter, using an anterior metal plate, the upper and lower vertebral body of the segment was fixated.

\section{Study methods and the assessment}

In regard to pre- and postoperative symptomatic improvement, pre- ad postoperative symptoms were evaluated based on the scale defined by Robinson. For the assessment of postoperative complications, an analysis was performed for complications that occurred preoperatively, postoperatively and at a final follow-up through interview and physical examination. From the first postoperative day, ambulation was not restricted. Measurement of cervical vertebral lordosis, defined as the angle between the second cervical vertebra (C2) lower margin and the sixth or seventh cervical vertebra (C6/7) lower margin, was based on lateral cervical radiographs taken preoperatively and at final follow-up. The lordosis curvature at final follow-up was measured and compared with that recorded preoperatively. We measured the distance between the upper and lower endplates at the sites of union on postoperative lateral radiograph of the cervical area. Subsequently, the degree of endplate subsidence was calculated by comparing with the value measured at final follow-up. Bone union was defined as cases in which mobility was absent on the lateral flexion and extension views of cervical radiographs. There was a bony bridge; and there was a bone shadow in a cage. Bone union was evaluated by calculating the union rate and the mean period elapsed until union was formed [5]. Statistical analysis was performed by using SPSS ver. 12.0 (SPSS Inc., Chicago, IL, USA). The Student t-test was used to examine for statistical significance in the postoperative period during which ambulation was regained, lordosis curvature changes occurred, the degree of end-plate subsidence and period of union. 


\section{Results}

The mean follow-up period was $24.7 \pm 6.42$ months (range, 18 to 38 months) in Group A and $16.8 \pm 3.23$ months (range, 12 to 28 months) in Group B. In Group A, the mean age was $54 \pm 9.72$ years (range, 26 to 76 years). The sex ratio (male : female) was $45: 14$ (0.76). There were 3 patients in their 20s (5\%), 3 patients in their 30s (5\%), 19 patients in their 40s (32\%), 15 patients in their 50s (25\%) and 19 patients in their 60s (32\%). In Group B, the mean age was $56 \pm 6.31$ years (range, 41 to 76 years). The sex ratio (male : female) was $14: 11(0.56)$. There were no patients in their $20 \mathrm{~s}(0 \%)$, no patients in their $30 \mathrm{~s}(0 \%), 10$ patients in their 40s (40\%), 11 patients in their 50s (44\%) and 4 patients in their $60 \mathrm{~s}(16 \%)$. There were significant differences in the mean follow-up period and sex ratio, but no significant difference in the mean age between the two groups (Table 1).

In regard to post-operative symptomatic improvement based on the Robinson scale, respectively 45, 10, and 4 patients experienced excellent, good and fair improvement in Group A. In Group B, respectively 19, 5, and 1 patients experienced excellent, good and fair improvement. In Group A, patients complained of post-operative pain at the donor site for autogenous bone. Otherwise, there were no notable complications in both groups. In Group B, however, there were 3 cases of incomplete union in which no bridge was formed either in the superior or the inferior region (one case of non-union in the superior region to the implant and two cases of that in the inferior region) within 12 months of surgery. All of these cases resulted in good clinical outcome. In delayed union or non-union, no poor clinical outcome was seen during a 12 to 18 month period postoperatively. The mean period during which ambulation could be performed following the operation was $5.4 \pm 1.8$ days (range, 3 to 7 days) in Group A and $2.1 \pm 0.5$ days (range, 1 to 4 days) in Group B. These differences reached statistical significance $(p=0.000)$. It is also assumed that difference in ambulation period arises from pain at the bone donor site. The mean curvature of final lordosis was increased by $8.3 \pm 2.4^{\circ}$ (range, 4 to $10^{\circ}$ ) in Group A and $4.7 \pm 1.2^{\circ}$ (range, 3 to $6^{\circ}$ ) in Group B. This difference also reached statistical significance $(p=0.000)$. The mean degree of subsidence in the endplate was $1.2 \pm 0.8 \mathrm{~mm}$ (range, 0 to $3 \mathrm{~mm}$ ) in Group A and $2.1 \pm 0.9 \mathrm{~mm}$ (range, 0

Table 1. Summary of cases

\begin{tabular}{lccc}
\hline \hline & Group A & Group B & $p$-value \\
\hline Mean follow-up period (mo) & $24.7 \pm 6.42(18-38)$ & $16.8 \pm 3.23(12-28)$ & 0.000 \\
Mean age (yr) & $54 \pm 9.72(26-76)$ & $56 \pm 6.31(41-76)$ & 0.073 \\
Sex ratio (male : female) & $45: 14$ & $14: 11$ & 0.001 \\
\hline
\end{tabular}
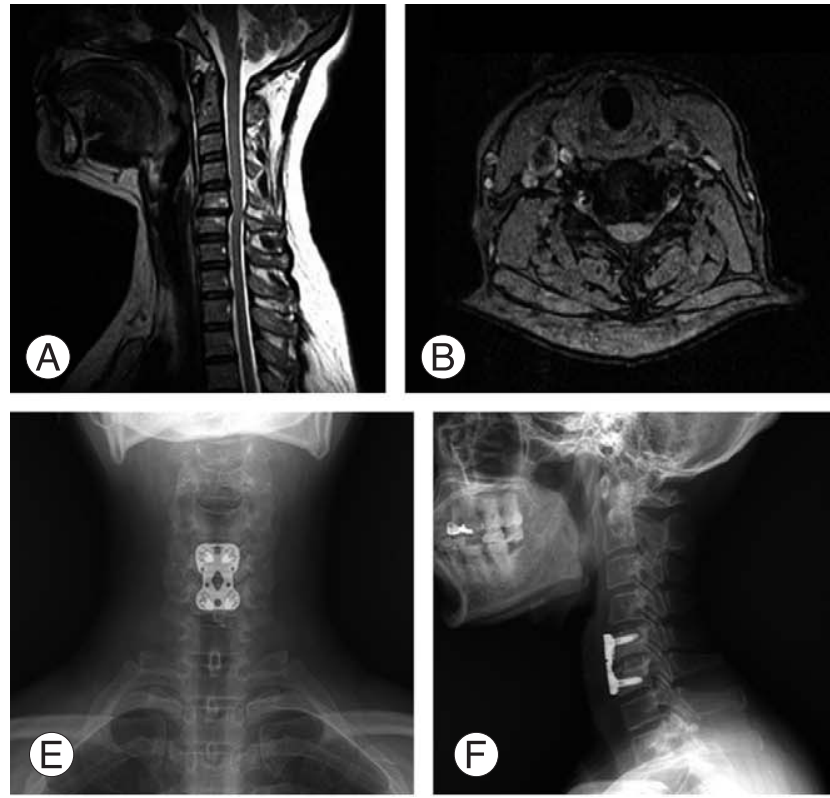
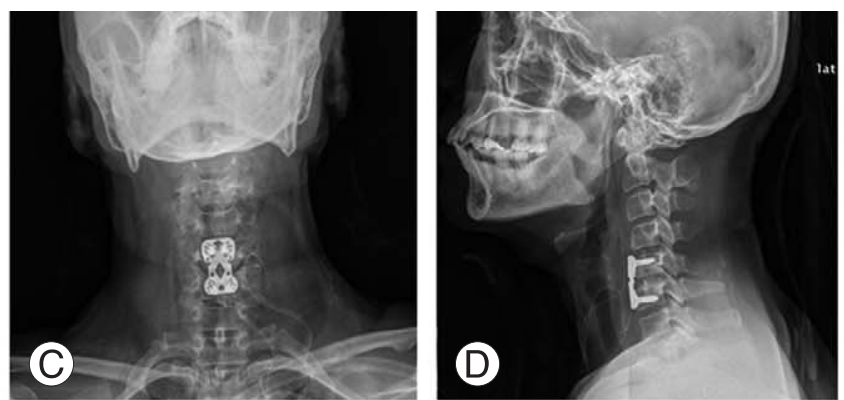

Fig. 1. A 45-year-old female diagnosed with spinal stenosis at C5-6. (A, B) Preoperative magnetic resonance imaging. (C, D) Postoperative radiographs. (E, F) Follow-up radiograph 12 months postoperatively. 
to $4 \mathrm{~mm}$ ) in Group B. This difference also reached statistical significance $(p=0.000)$. Bone union occurred at rate of $88 \%$ in Group B, whereas all patients experienced bone union in Group A (union rate: 100\%) (Fig. 1). The mean period of bone union was $13.3 \pm 3.5$ weeks (range, 12 to 24 weeks) in Group A and $46.6 \pm 8.7$ weeks (range, 24 to 52 weeks) in Group B (Figs. 2 and 3). This difference also reached statistical significance $(p=0.000)$ (Table 2).
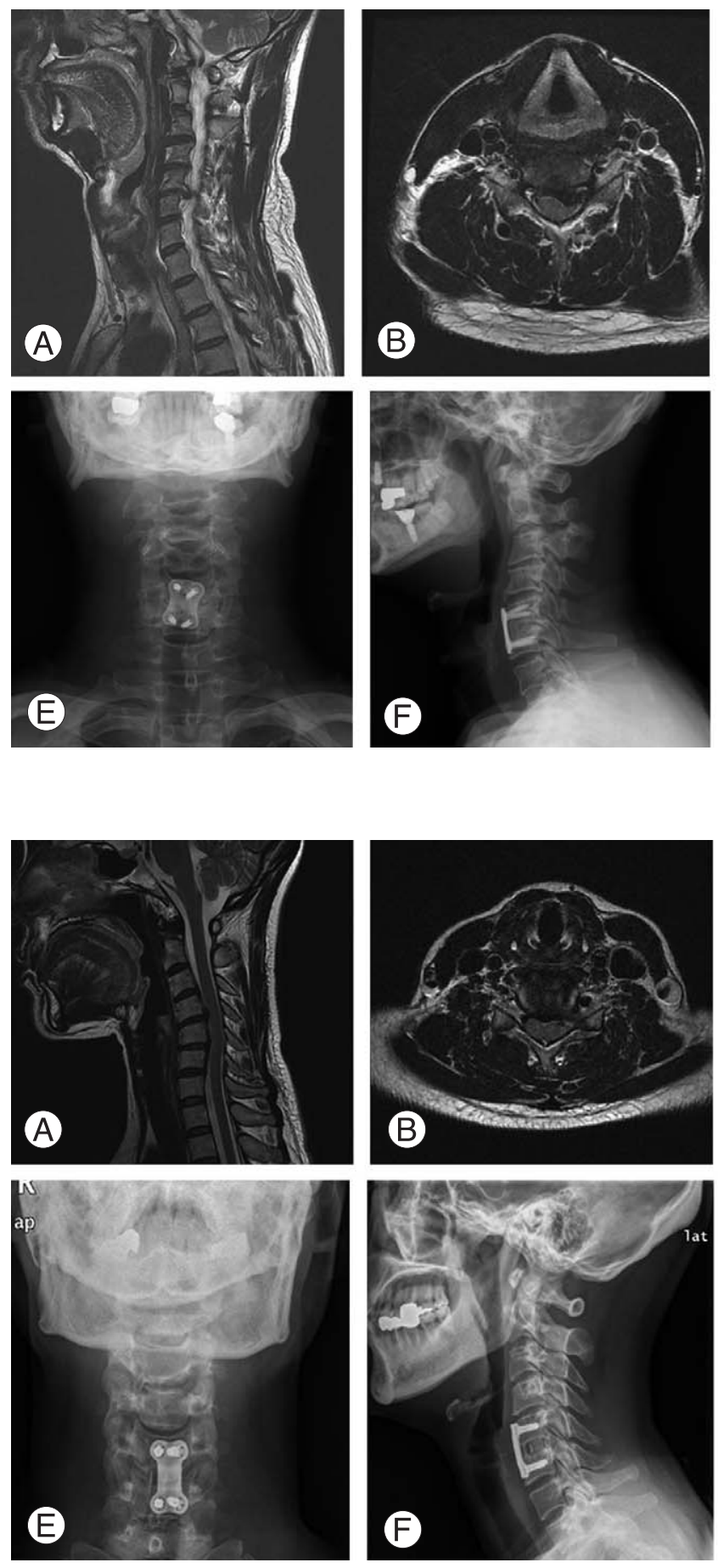
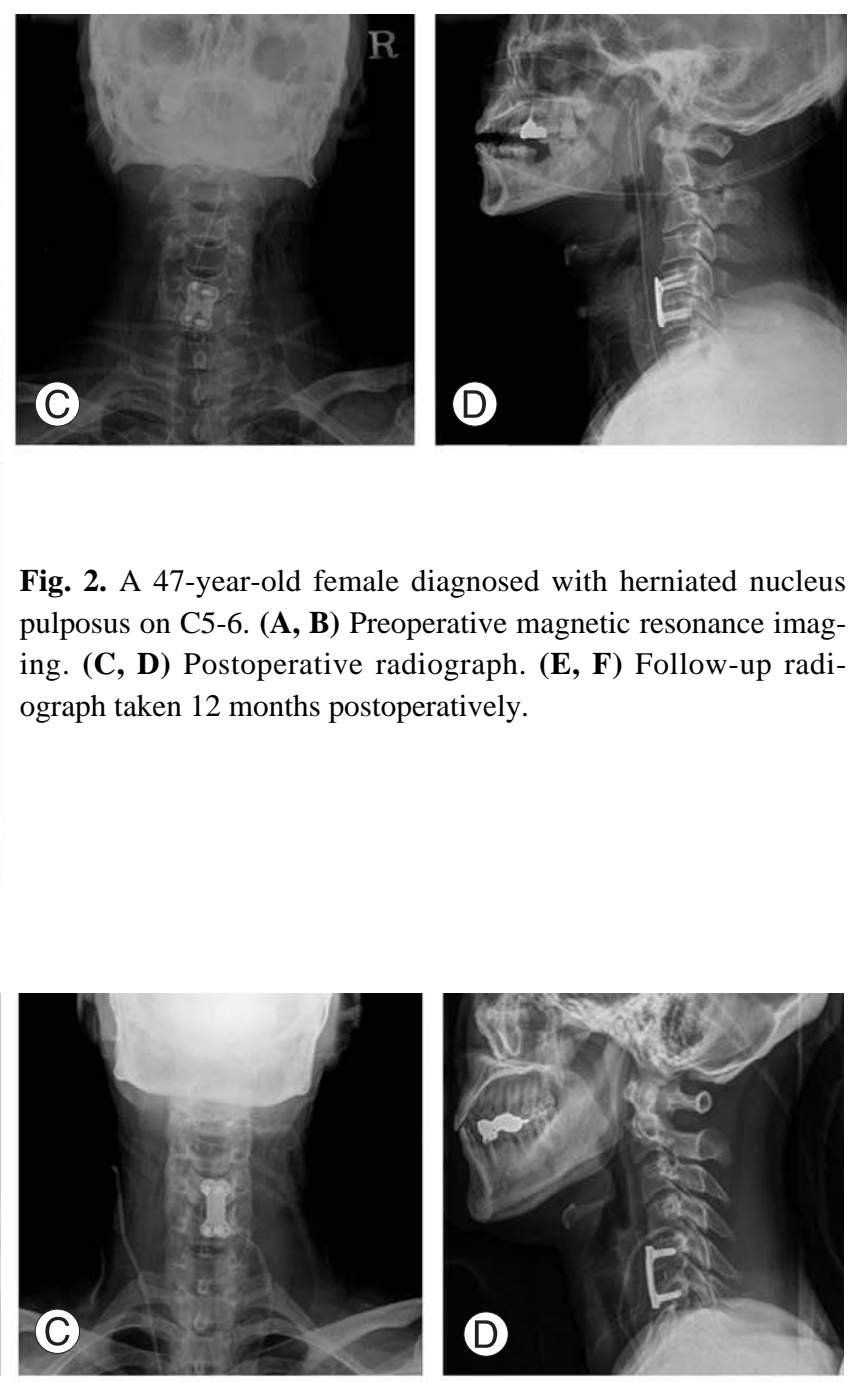

Table 2. Results of study

\begin{tabular}{lrrc}
\hline \hline & Group A & Group B & $p$-value \\
\hline Ambulation time (day) & 5.4 & 2.1 & 0.000 \\
Lordosis increase $\left(^{\circ}\right.$ ) & 8.3 & 4.7 & 0.000 \\
Collapse or subsidence (mm) & 1.2 & 2.1 & 0.000 \\
Union period (wk) & 13.3 & 46.6 & 0.000 \\
\hline
\end{tabular}

Fig. 2. A 47-year-old female diagnosed with herniated nucleus pulposus on C5-6. (A, B) Preoperative magnetic resonance imaging. (C, D) Postoperative radiograph. (E, F) Follow-up radiograph taken 12 months postoperatively.

Fig. 3. A 50-year-old female diagnosed with spinal stenosis on C5-6. (A, B) Preoperative magnetic resonance imaging. (C, D) Postoperative radiograph. (E, F) Follow-up radiograph taken 12 months postoperatively. 


\section{Discussion}

It is known that anterior cervical disectomy and fusion are being used most frequently to treat trauma and diseases in the cervical area [1-4]. Currently, Smith-Robinson's method [1] for intervertebral bone graft is being adopted most commonly. The complication rates have been reported to be $9.4-49 \%$ across all methods based on bone graft [6]. The complications include pain, hematoma, infection, lateral femoral cutaneous nerve injury, ilium fracture, peritoneal perforation, hernia, and cosmetic problems [7,8]. According to Sawin et al. [9] in patients who experienced postoperative pain at the donor site for autogenous bone graft, $36 \%$ of patients continued to experience pain one year postoperatively. Even within four years postoperatively, $31 \%$ of patients experience pain at the donor site. In the current study, in the group where the autogenous bone was grafted, patients complained of pain occurring at the donor site. In comparison with patients who received a cage, patients who received autogenous bone grafts took significantly longer to ambulate. To avoid postoperative complications that may occur while bone samples are harvested, methods that entail a cage and alternative bone substitute to bone graft have been introduced.

The cage and bone substitute methods enjoy advantages that include reduced bleeding, operation time and skin scars during harvesting of bone graft materials and there is no need for postoperative orthosis. Thus, early ambulation can be achieved and prompt return to daily lives is possible. At our institution, the use of orthosis is recommended for approximately six weeks in patients who received autogenous bone graft and for up to three days in patients who received a cage.

In the current study, a tricalcium phosphate (TCP) cage was used. TCP has osteogenic potential comparable to that of hydroxyapatite. It has also been reported to produce excellent treatment outcomes in implant denaturation [10]. According to Xie et al. [11], TCP has a higher degree of absorptivity compared with hydroxyapatite. It is therefore replaced more promptly by new bones. However, there are also disadvantages, such as lower degree of mechanical rigidity compared with hydroxyapatite. In patients who received a TCP cage, as shown in the current study, the period of bone union was prolonged due to a lack of osteoinductibity. The disadvantages of TCP include rupture and extrusion of cage and cage endplate subsidence.
According to Zulkefli et al. [12] the union rate at 6-month follow-up was $95 \%$ in patients who received autogenous bone graft, and $62.1 \%$ in patients who received a hydroxyapatite cage. It has been reported, however, that the rate of bone union at final follow-up is close to $100 \%$ and the rate of clinical success exceeds 90\% [13-15]. In the current study, the mean period of union was prolonged by approximately 33 weeks in patients who received a cage. In three patients, no union was seen during the follow-up period (at a 12-, 15- and 18-month follow-up, respectively) and there were no notable clinical complications. It is envisaged that continual follow-up would be needed until bone union is achieved.

According to Kettler et al. [16] and Wilke et al. [17] subsidence in the cage endplate arises from instability due to mobility of the cervical area following disectomy. Subsidence is variable with cage endplate design. Compared with bone segment, however, subsidence has been reported to occur at higher incidence. Van Jonbergen et al. [18] reported that subsidence in the endplate occurred at a mean length of $1.06 \mathrm{~mm}$ during a 6-month follow-up period. In addition, Schmieder et al. [19] also reported that it occurred at a mean length of $1.13 \mathrm{~mm}$ in the anterior region, and $0.9 \mathrm{~mm}$ in the posterior region at 2-year follow-up. Reports suggest that endplate subsidence significantly retains increased intervertebral disc space compared with that preoperatively. It is able to improve the overall sagittal alignment of the cervical vertebrae, and its severity and incidence have no effects on clinical outcomes. In the current study, the mean endplate subsidence was increased by approximately $1 \mathrm{~mm}$ in patients who receive a cage compared with patients who received autogenous bone graft. This difference reached statistical significance. Because the period of bone union was prolonged in patients who received a cage, it is assumed that the above results may be related to longer exposure to instability due to cervical movement.

Our results also showed that the curvature of lordosis was increased in both patient groups $\left(8.3^{\circ}\right.$ in Group A vs. $4.7^{\circ}$ in Group B). There was significant difference between both groups. These results suggest that there is an advantage in forming curvature based on the surgeon's judgment compared with patients in whom a cage was used of curved type by using a wedge shape struct bone for the autogenous bone graft. It is also presumed that the endplate subsidence, presumably occurred as a result of delayed bone union, may be a contributory factor. However, there was no significant difference in clinical symptoms between the two groups. In the 
current study, however, there was significant difference in endplate subsidence and curvature on radiography performed at final follow-up in 7 Group A patients and 4 Group B patients. It is therefore assumed that continuous follow-up should be performed until there are no changes in these parameters at two consecutive follow-ups in the 11 aforementioned patients.

\section{Conclusions}

In patients who underwent anterior cervical disectomy and fusion for the management of trauma and diseases in the cervical area, changes in lordosis curvature and bone union period following operation were better in those who received autogenous bone graft, compared with those who received a cage containing bone substitute. The former patient group experienced less pain at the autogenous bone graft donor site and ambulation was possible.

Each patient group enjoys some advantages. At final follow-up, the clinical outcomes were satisfactory. There was no significant difference between the clinical outcomes of both groups, except in ambulatory time, mean curvature of final lordosis, endplate subsidence and period of bone union. In the current study, the number of patients who received a cage containing bone substitute was smaller than the number of patients who received autogenous bone graft. Besides, the current study is limited by short-term followup. This will require further investigations.

\section{REFERENCES}

1. Robinson RA, Smith GW. Anterolateral cervical disc removal and interbody fusion for cervical disc syndrome. Bull Johns Hopkins Hosp 1955;96:223-4.

2. Aebi M, Zuber K, Marchesi D. Treatment of cervical spine injuries with anterior plating. Indications, techniques, and results. Spine (Phila Pa 1976) 1991;16(3 Suppl):S38-45.

3. Farey ID, McAfee PC, Davis RF, Long DM. Pseudarthrosis of the cervical spine after anterior arthrodesis: treatment by posterior nerve-root decompression, stabilization, and arthrodesis. J Bone Joint Surg Am 1990;72:1171-7.

4. Gore DR, Sepic SB. Anterior cervical fusion for degenerated or protruded discs: a review of one hundred forty-six patients. Spine (Phila Pa 1976) 1984;9:667-71.

5. Grob D, Peyer JV, Dvorak J. The use of plate fixation in anterior surgery of the degenerative cervical spine: a comparative prospective clinical study. Eur Spine J 2001;10:
408-13.

6. Banwart JC, Asher MA, Hassanein RS. Iliac crest bone graft harvest donor site morbidity: a statistical evaluation. Spine (Phila Pa 1976) 1995;20:1055-60.

7. Kurz LT, Garfin SR, Booth RE Jr. Harvesting autogenous iliac bone grafts: a review of complications and techniques. Spine (Phila Pa 1976) 1989;14:1324-31.

8. Kurz LT, Garfin SR, Booth RE Jr. Iliac bone grafting: techniques and complications of harvesting. In: Garfin SR, editor. Complications of spine surgery. Baltimore: Williams and Wilkins; 1989. p. 323-41.

9. Sawin PD, Traynelis VC, Menezes AH. A comparative analysis of fusion rates and donor-site morbidity for autogeneic rib and iliac crest bone grafts in posterior cervical fusions. J Neurosurg 1998;88:255-65.

10. Shimazaki K, Mooney V. Comparative study of porous hydroxyapatite and tricalcium phosphate as bone substitute. J Orthop Res 1985;3:301-10.

11. Xie Y, Chopin D, Hardouin P, Lu J. Clinical, radiological and histological study of the failure of cervical interbody fusions with bone substitutes. Eur Spine J 2006;15:1196203.

12. Zulkefli A, Jeyasilan K, Zairul AK, Ramanathan R. Comparison of fusion rate between autogenous tricortical iliac crest bone graft and hydroxyapatite block graft in anterior cervical discectomy and fusion surgery. Malaysian Orthop J 2009;3:36-9.

13. Coric D, Branch CL Jr, Jenkins JD. Revision of anterior cervical pseudoarthrosis with anterior allograft fusion and plating. J Neurosurg 1997;86:969-74.

14. Savolainen S, Rinne J, Hernesniemi J. A prospective randomized study of anterior single-level cervical disc operations with long-term follow-up: surgical fusion is unnecessary. Neurosurgery 1998;43:51-5.

15. Shapiro S. Banked fibula and the locking anterior cervical plate in anterior cervical fusions following cervical discectomy. J Neurosurg 1996;84:161-5.

16. Kettler A, Wilke HJ, Claes L. Effects of neck movements on stability and subsidence in cervical interbody fusion: an in vitro study. J Neurosurg 2001;94(1 Suppl):97-107.

17. Wilke HJ, Kettler A, Goetz C, Claes L. Subsidence resulting from simulated postoperative neck movements: an in vitro investigation with a new cervical fusion cage. Spine (Phila Pa 1976) 2000;25:2762-70.

18. van Jonbergen HP, Spruit M, Anderson PG, Pavlov PW. Anterior cervical interbody fusion with a titanium box cage: early radiological assessment of fusion and subsi- 
dence. Spine J 2005;5:645-9.

19. Schmieder K, Schönmayr R, Goetz C, et al. Anterior cervical interbody fusion with Wing titanium cages. In: Kaech DL, Jinkins JR, editors. Spinal restabilization procedures: diagnostic and therapeutic aspects of intervertebral fusion cages, artificial discs and mobile implants. Boston: Elsevier; 2002. p. 229-34. 